\title{
EMITTANCE MEASUREMENTS OF THE SPACE CHARGE DOMINATED THOMSON SOURCE PHOTOINJECTOR
}

\author{
S. G. Anderson, J. B. Rosenzweig, UCLA, Los Angeles, CA 90095, USA \\ G. P. LeSage, J. Crane, LLNL, Livermore, CA
}

\begin{abstract}
The photocathode rf gun test facility in Livermore National Laboratory's Thomson source is described. The quadrupole scan and slit based emittance measurement techniques are used to measure the emittance of the space charge dominated beam. Experimental results are compared with simulation and theoretical calculations.
\end{abstract}

\section{INTRODUCTION}

The modern radio frequency (rf) photoinjector is a source that produces very high brightness electron beams. [1] It does so by generating a high charge bunch with the same temporal structure and transverse quality as the driving picosecond scale laser pulse. Photoinjector beams generate extremely large spacecharge forces and care must be taken in order to preserve the brightness of the beam despite these forces. Longitudinally, this is accomplished by applying large amplitude rf accelerating fields. Transversely, the phase space density of the beam is preserved by balancing the space-charge defocusing and externally applied focusing forces. This process in called emittance compensation, and it has been studied in detail experimentally [2] and theoretically. [3,4]

The space-charge dominated behavior of photoinjector beams must be taken into account in the measurement of their properties as well as in accelerating them. In addition, issues related to short pulse lengths and drive laser fluctuations must be addressed for many types of measurements.

In this paper, we examine the measurement of the transverse emittance of a photoinjector beam. The Thomson source photoinjector [5] at Lawrence Livermore National Laboratory was used to measure the transverse emittance of the beam with the quadrupole scan and slit based measurement techniques. The Thomson source was chosen to do these measurements because it offers a short-pulse, low energy, $(5 \mathrm{MeV})$ highly space-charge dominated beam. As discussed below, the results of the measurements show that the quadrupole scan method gives a higher value of emittance than the slit based technique.

\section{EMITTANCE MEASUREMENT TECHNIQUES}

The two methods used to measure emittance in this experiment, the quad scan and the slit system, are described here.

\subsection{Quadrupole Scanning}

The quadrupole scanning measurement technique is widely used in the accelerator physics community. [6] A description of the process is given here in order to show it's limitations when applied to space-charge dominated beams.

The measurement can be understood by examining the evolution of a beams rms Twiss parameters in a drift length following a thin lens (of focal length $\mathrm{f}$.)

$$
\begin{gathered}
\beta_{x}^{\prime}=2 \frac{\left\langle x x^{\prime}\right\rangle}{\varepsilon_{x}}=-2 \alpha_{x} \\
\alpha_{x}^{\prime}=-\frac{\left\langle x^{\prime 2}\right\rangle+\left\langle x x^{\prime \prime}\right\rangle}{\varepsilon_{x}}=-\gamma_{x} \\
\gamma_{x}^{\prime}=2 \frac{\left\langle x^{\prime} x^{\prime \prime}\right\rangle}{\varepsilon_{x}}=0
\end{gathered}
$$

Here we have explicitly ignored space-charge and externally applied forces to set $x^{\prime \prime}=0$. By applying initial conditions to equation (1), we can solve the equation for the $\beta$-function in terms of the square of the rms beam size as:

$$
\begin{aligned}
& \sigma_{x}^{2}(z)=\left[\sigma_{x 0}^{2}-2 \alpha_{x 0} \varepsilon_{x}\left(z-z_{0}\right)+\gamma_{x 0}\left(z-z_{0}\right)^{2}\right]+ \\
& \frac{2 \sigma_{x 0}^{2}}{f}\left[\frac{\alpha_{x 0}}{\beta_{x 0}}\left(z-z_{0}\right)^{2}-\left(z-z_{0}\right)\right]+\frac{\sigma_{x 0}^{2}}{f^{2}}\left(z-z_{0}\right)^{2}
\end{aligned}
$$

In this form, we see that the square of the beam size at the end of the drift should follow the square of the focusing strength of the lens. The quad scan procedure then, is to vary to current in a quadrupole magnet and measure the beam size at a fixed distance from the lens.

\subsection{Emittance Slits}

In this measurement system [7], the beam is collimated by a set of slits. The beamlets produced by the slits then drift a fixed length to an intercepting diagnostic where the intensity profile of the beamlets can be measured. 
The slits actually serve a dual purpose. The first is to provide a way to extract the horizontal phase space of the original beam. Since the slit separation is known, the number of slits illuminated gives the size of the beam on the slits. The position and width of each beamlet on the screen combined with the length of the drift give the beamlets average momentum and momentum spread.

The second function of the slits is to make the beamlets emittance dominated. That is, to create beamlets that expand in the drift space because of their thermal emittance, and not due to space charge forces within the beamlet. The relative strength of these two effects can be seen by taking the ratio of the two forcing terms in the rms envelope equation.

$$
\sigma_{x}^{\prime \prime}=\frac{\varepsilon_{n}^{2}}{\gamma^{2} \sigma_{x}^{3}}+\frac{2 I}{\gamma^{3} I_{0}\left(\sigma_{x}+\sigma_{y}\right)}
$$

For the full beam this ratio is:

$$
R=\frac{I \sigma_{0}^{2}}{I_{0} \gamma \varepsilon_{n}^{2}}
$$

For beams in this experiment, $R \approx 50$, indicating a space-charge dominated beam. For the beamlets however, this ratio becomes:

$$
R_{\text {beamlet }}=\sqrt{\frac{2}{3 \pi}} \frac{I}{\gamma_{0}}\left(\frac{d}{\varepsilon_{n}}\right)^{2}
$$

Where $\mathrm{d}$ is the slit width $(50 \mu \mathrm{m}$ in this case.) This ratio is typically on the order of $10^{-2}$, indicating emittance dominated beamlets.

\section{EXPERIMENTAL PROCEDURE}

The beamline configuration used for these measurements is shown in figure 1. The accelerator in this setup was a 1.6 cell, S-band, BNL-SLAC-UCLALLNL rf photo-cathode gun [8]. The gun produced a 5 $\mathrm{MeV}$ beam. An emittance compensating solenoid after the gun was used to control the rf defocusing at the gun exit and allowed us to select a reasonable beam size at the emittance slits and quadrupole. The magnetic field at the cathode was nulled with an identical bucking solenoid placed upstream of the cathode. The charge was measured using an integrating current transformer (ICT.) The laser injection phase was monitored during the experiments by mixing the low level rf derived from the laser oscillator with that from a probe in the gun full cell. Long time-scale drift in the rf was corrected by hand for all measurements with a manual phase shifter.

The photocathode drive laser system consists of a fiber-seeded regenerative amplifier, a multipass power amplifier, a pulse compressor, and frequency conversion crystals for frequency-tripling the $800 \mathrm{~nm}$ laser pulse. The two laser amplifiers, regen and multipass, are pumped by a single, frequency-doubled, Q-switched YAG laser that puts out $300 \mathrm{~mJ}$ of $532 \mathrm{~nm}$ light in a $8 \mathrm{~ns}$ pulse. The output beam of the multipass amplifier is expanded and sent to the grating compressor.

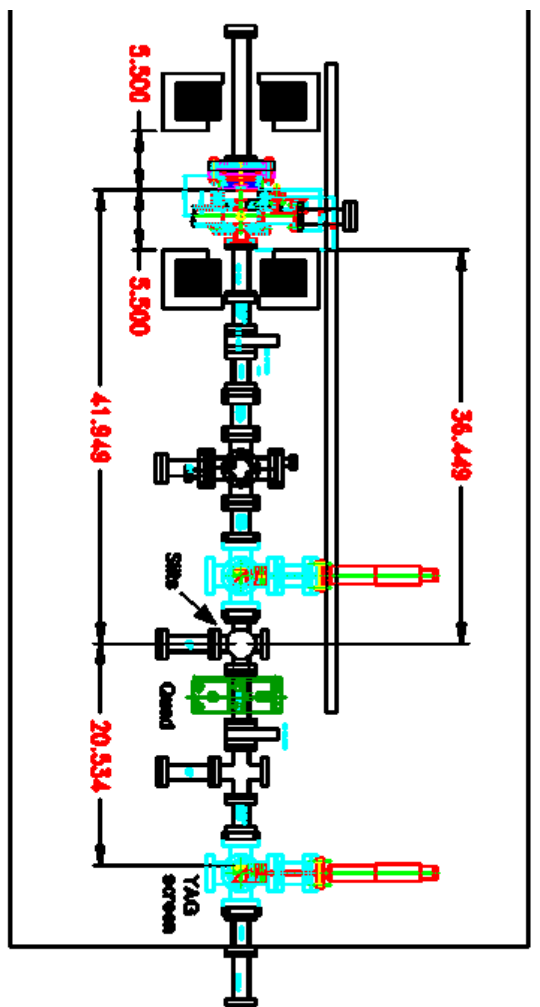

Figure 1: Experimental Apparatus

Since we wished to explore the differences between the two measurement techniques as the space-charge forces varied, we chose to take measurements with both the slits and the quad scan at several different values of beam density. This was done by changing the compressor grating separation. At a given separation, the dimensions of the laser and electron beams as well as the charge and injection phase were measured. This allowed us to calculate the plasma frequency of the beam in order to compare our measurements with simulation and analytical models. The range of beam parameters used in these measurements is given in table 1.

\begin{tabular}{||l|l||}
\hline PARAMETER & RANGE \\
\hline \hline Electron Beam Charge & $50-300 \mathrm{pC}$ \\
\hline RMS laser spot size & $0.5-2 \mathrm{~mm}$ \\
\hline RMS laser pulse length & $2.5-6 \mathrm{psec}$ \\
\hline
\end{tabular}

Table 1: Electron Bunch Parameters

\section{RESULTS}

The result of the emittance measurements is shown in figure 2. Here we plot the measured emittance as a function of the beam plasma wave number at the quadrupole times the drift length. In addition, simulations of the quad scan (described below) are included in the figure. 


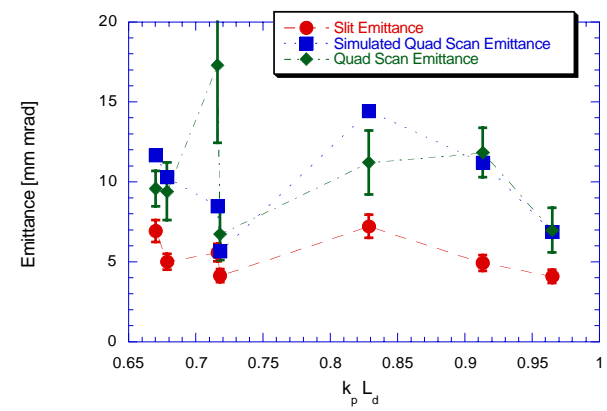

Figure 2: Emittance measurements from the slits, (red circles) the quad scan, (green diamonds) and the simulation of a quad scan (blue squares).

Figure 2 shows that the slit measurements give approximately a factor of two lower values than the quad scan.

\section{ANALYSIS}

In analyzing the emittance data we began by simulating the photoinjector up to the position of the quadrupole using PARMELA [9] for each of the charges and laser pulses in the experiment. The emittances given by PARMELA were about $4 \mathrm{~mm} \mathrm{mrad}$, which agrees reasonably with the slit measurements. The next step was to simulate the process of the quad scan with spacecharge taken into account. This was done in three different ways, using PARMELA with point-to-point space-charge calculations, using HOMDYN [10], and by numerically integrating equation 3 (and it analog in the y-dimension.) In each simulation the emittance of the beam was taken to be the value obtained in the corresponding slit measurement. The results of the HOMDYN simulations are plotted in figure 2, and we see that they follow the quad scan measurements reasonably well.

In beginning a theoretical treatment of the quad scan with space-charge, one may consider working with equation 3 , but ignoring the emittance term. In this experiment however, both terms are relevant. The beam waist is, in fact, emittance dominated, but the waist position and depth are effected by the space-charge dominated behavior of the beam prior to a waist.

We can gain an insight into the significance of spacecharge in a quadrupole scan by rewriting the envelope equation in terms of the plasma wave number.

$$
\sigma_{x}^{\prime \prime}=\frac{\varepsilon_{n}^{2}}{\gamma^{2} \sigma_{x}^{3}}+\frac{k_{p}^{2} \sigma_{x}}{2}
$$

If we ignore the emittance term here, the solution is $\sigma_{x} \propto \cosh \left(k_{p} z\right)$. This gives us a reasonable rule of thumb for the quad scan: space charge is an important factor when $k_{p} L_{\text {drift }} \approx 1$. This rule applies in our measurement, as we see that both the data and simulation indicate a larger emittance than predicted by PARMELA and measured with the slits.

\section{REFERENCES}

[1] P. O'Shea, Proc. $2^{\text {nd }}$ ICFA Advanced Accelerator Workshop on the Physics of High Brightness Beams, 17 (World Scientific, 2000).

[2] X. Qiu et al., Phys. Rev. Lett. 76, 3723 (1996).

[3] B. E. Carlsten, Nucl. Inst. Methods A 285, 313 (1989).

[4] Luca Serafini and J. B. Rosenzweig, Phys. Rev. E 55, 7565 (1997).

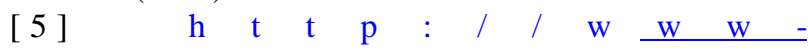
phys.llnl.gov/Organization/HDivision/Research/LIN AC/LINACFacilityVirtualTour/photoinjector.html

[6] J. T. Seeman, Handbook of Accelerator Physics and Engineering, 559, Chao and Tigner (1999).

[7] http://pbpl.physics.ucla.edu/papers/PDF/emitslit.pdf

[8] D. T. Palmer, et al., Proc. 1997 Particle Accelerator Conf., 2846 (IEEE, 1997).

[9] http://pbpl.physics.ucla.edu/docs/parmela.pdf

[10] M. Ferrario, et al., Proc. $2^{\text {nd }}$ ICFA Advanced Accelerator Workshop on the Physics of High Brightness Beams, 534 (World Scientific, 2000). 